\title{
P-94
}

\section{Anti-Inflammatory, Antinociceptive and Antipyretic Investigation of Catechin - Like Compound from Partial Purification of Dichloromethane Crude Extracts from leaves of Labisia Pumila}

\author{
Patrick N. Okechukwu ${ }^{1,}$, Sopia O. Ekeuku ${ }^{1}$, Samuagam Loshnie ${ }^{1}$ and Gaberiel A. Akowuah ${ }^{2}$ \\ ${ }^{, I}$ Biotechnology program, Faculty of Applied Sciences, ${ }^{2}$ School of Pharmacy, Faculty of Pharmaceutical Sciences, UCSI \\ University, No. 1, Jalan Menara Gading, UCSI Heights, 56000 Kuala Lumpur, Malaysia.Email: patrickn@ucsi.edu.my
}

Labisia pumila, LP (Myrsinaceae), is a popular herb among the women in Malaysia known locally as "Kacip Fatimah. It has been claimed by the traditional medicinal practitioner as a pain reliever, treat inflammatory disorders, such as rheumatism and fever. This study was aimed to investigate the partially purified crude extracts of Labisia pumila (DELP) for its antiinflammatory, antinociceptive and antipyretic effects. Acetic acid-induced abdominal writhing and carrageenan-induced paw oedema was used to evaluate the anti-inflammatory activity whereas hot plate test and formalin test was used for the antinocicpetive activity. Antipyretic activity was determined by yeast induced pyrexia method. Partial purification of DELP extract resulted in 5 different fractions (A-E). All the fractions of DELP showed significant anti-inflammatory, antinociceptive and antipyretic activity in the entire test used. Phytochemical screening revealed the presence of flavonoids, steroids, saponin, alkaloids and tannins. HPLC analysis revealed the presence of catechin, The present study confirmed that the extracts exhibited anti-inflammatory, antinociceptive and antipyretic activity, supporting its folk use and is in consonances with our earlier report.

Keywords: Anti-inflammation, Antinociception, Antipyretic, Labisia pumila, phytochemical screening. 